Short Communications

\title{
MATRIX BUTTON PROVISIONALS
}

\author{
Vinaya Bhat \\ Professor, Department of Prosthodontics \\ A.B. Shetty M emorial Institute of Dental Sciences, Deralakatte, Mangalore - 575 018, Karnataka \\ Mobile No. : $9481921180 \quad$ E-mail : drvinayabhat@gmail.com
}

\begin{abstract}
:
Fabrication of provisional restorations has been explained using different materials and techniques in the past. Formation of an external matrix before preparation of the abutment, which is later used in combination with a resin material, is one of the techniques. Here, a new material has been used as matrix in direct, indirect and indirect-direct methods to produce esthetic and functional provisional restorations. There is neither wasting of chair side time nor a second visit for the patient.
\end{abstract}

Keywords: Provisional Restorations, External Surface Form, Thermoplastic, Matrix

\section{MATRIXBUTTON PROVISIONALS}

\section{Introduction}

Provisional Restorations are an integral part of fixed prosthodontic treatment. Malone et al ${ }^{1}$ named them as "treatment restorations" or "Healing Matrix" and explained various metallic and non-metallic materials for their fabrication. For non-metallic materials there are a variety of techniques available to suit the individual needs of the clinician and the situation, whether it is an inlay, single crown, bridge, complete arch restoration or even implant. ${ }^{1.5}$

M alone et $\mathrm{al}^{1}$, Rosenstiel et $\mathrm{al}^{6}$ and Dykema et al ${ }^{7}$ suggest a method to form a mold cavity or an External Surface Form, into which a plastic material is poured and the internal or the tissue surface form is fabricated. For creating external surface form, an alginate impression, base plate wax impression, silicone putty impression, polycarbonate preformed shell, or custom preformed Fixed Partial Denture (FPD) shells can be used. They can also be custom fabricated using a thermoplastic sheet, which is heated and adapted to a stone cast with vacuum or air pressure while the material is still pliable. This requires an electric heating element and a vacuum source.

The purpose of this article is to present a new thermoplastic material that does not require elaborate equipment for its manipulation. It is a circular disc of thickness about 2 millimeters and diameter 22 millimeters. ${ }^{8}$ The softening temperature is around 65 $70^{C}$ and the softening time is approximately 45 seconds. There is a change in color from white to clear when the disc is completely soft and ready to be kneaded. (Figure 01) Working time is around 5 minutes.

Provisional restorations can be fabricated using any one of the following techniques:

\section{Techniques:}

\section{INDIRECT}

1. Diagnostic casts of the entire mouth are required for this method. On the casts, make all the modifications as per the case. For the edentulous area, an artificial tooth may be arranged in wax, corresponding to the patient's occlusion. (Figure 02)

2. The matrix is formed on the cast. More than one disc can be softened and kneaded according to the requirement. Place discs in hot water bath at $65-70^{c}$ in a clean rubber bowl or ceramic cup. In approximately 45 seconds discs change their color from white to clear. (See Figure 01)

3. Pick up the soft discs using tweezers and knead them to form an oblong mass.

4. Press it down on the occlusal surfaces of the teeth on the cast and mold it buccally, lingually and into the 
undercuts. (Figure 03)

5. $M$ ark the buccal side with explorer for reference while reseating later. Cool the matrix with air syringe for 30 seconds and watch the color revert back to white! Remove the matrix and examine for completeness. (Figure 04)

6. After the abutments are prepared, an impression is made with irreversible hydrocolloid. Cast is poured with quick setting stone.

7. Cast is retrieved from the impression. Then the matrix is filled with the selected provisional material (Figure 05-a) and it is placed over the cast with prepared abutments. A rubber band can be used to hold the matrix and the cast together while the material is polymerizing. (Figure $05-b$ )

8. Once the material is set remove the matrix from the preparation and peel the matrix away. (Figure 06) Trim the excess material (Figure 07), polish and lute the restoration with suitable cement.

9. There are several materials that can be used with this matrix for the fabrication of provisionals. Ethylacrylates, auto mix bisacryls or light cure resins may be used and the matrix is seated on the prepared tooth. When using acrylics, it is advisable to coat the internal surface of the matrix lightly with separating medium. Remove the crown in rubbery phase or wait till final set, lifting it on and off the preparation.

II DIRECT(for small restorations)

1. Anesthetize the tooth concerned. (Not required if non-vital). While the anesthesia is taking up, soften the disc and form the matrix directly in the mouth.

2. Complete the abutment preparation and try the matrix on to check for accurate seating.

3. Fill the matrix with required material, apply petrolatum on the tooth and soft tissue around. Place the loaded matrix on the prepared tooth. Remove the matrix when the material reaches rubbery phase, lift it on and off the preparation.
4. Once it is set, remove the matrix from the preparation and peel the matrix away. Trim the excess material, polish and lute the crown with suitable cement.

\section{INDIRECT-DIRECT}

1. In this method at least the diagnostic cast of the arch concerned is required. If the cast of the opposing arch available, the provisional restoration would be made to respect the occlusion.

2. The pontic area/s are replaced with artificial tooth/teeth fixed firmly in wax.

3. The matrix is formed on the cast as explained earlier utilizing one or more discs.

4. After the abutments are prepared, fill the matrix with the material of choice and place it on the abutments. Allow the material to completely set. Trim, adjust the occlusion if required, polish and cement the restoration.

\section{Summary}

A new matrix material used for the fabrication of provisional restoration has been explained. The advantages of the technique include, accurate reproduction of the contours of the tooth satisfying esthetics and occlusal demands of the treatment. Density of the plastic drives the crown material into the margins extremely well.

The thermoplastic material is easy to handle, requires no extra armamentarium other than what is available in any dental office, quick to manipulate when soft and mold on to the tooth. It has sufficient working time for kneading and adaptation on the teeth or the cast. In a busy practice, it saves valuable chair side time for the dentist.

The crown material can be completely light cured through them. The matrix can be saved along with other information in the event patients lose or break their provisionals. Several discs can be softened together and molded to form a larger mass for multiple unit restorations. 


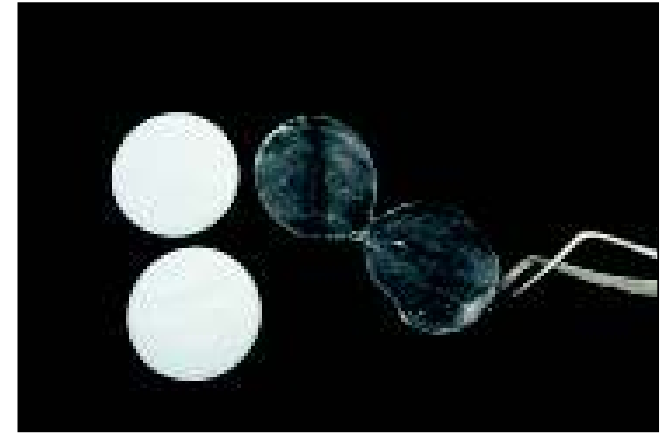

M atrix button, before and after softening

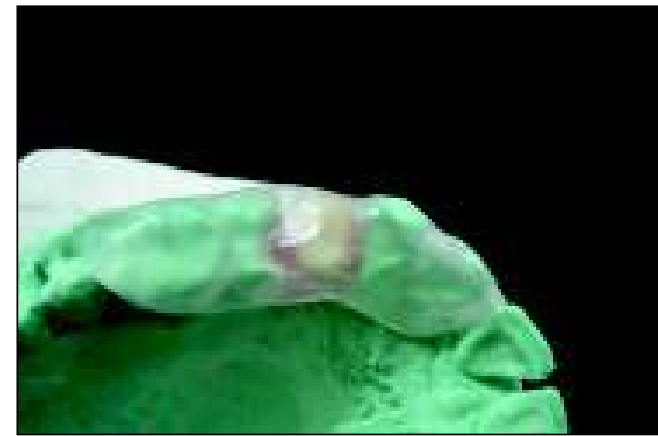

Molded M atrix buttons

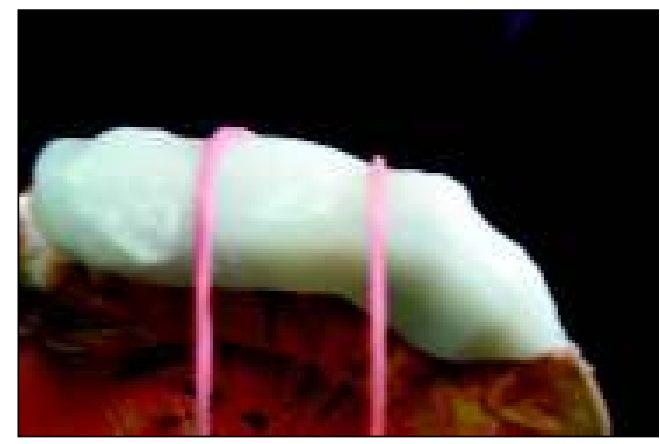

M atrix loaded with provisional material

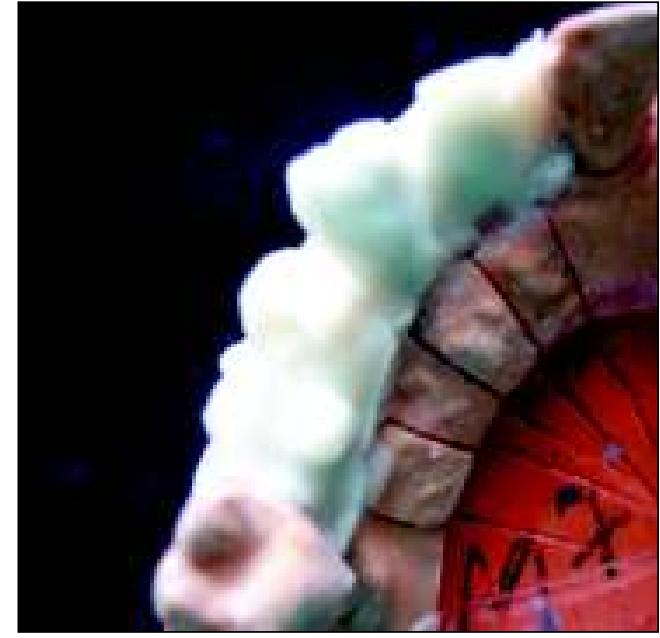

Provisional restoration - before trimming

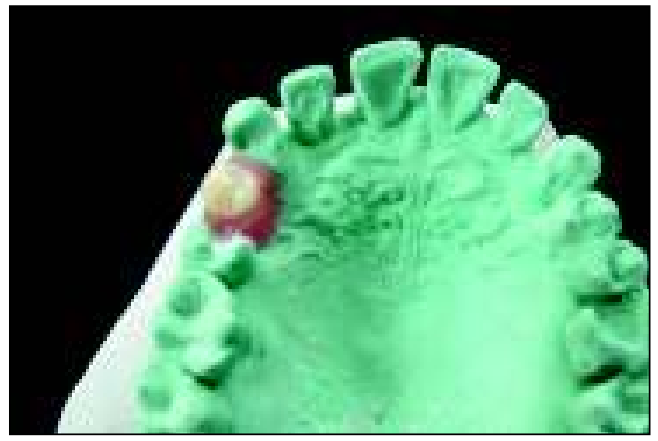

Modified Diagnostic cast

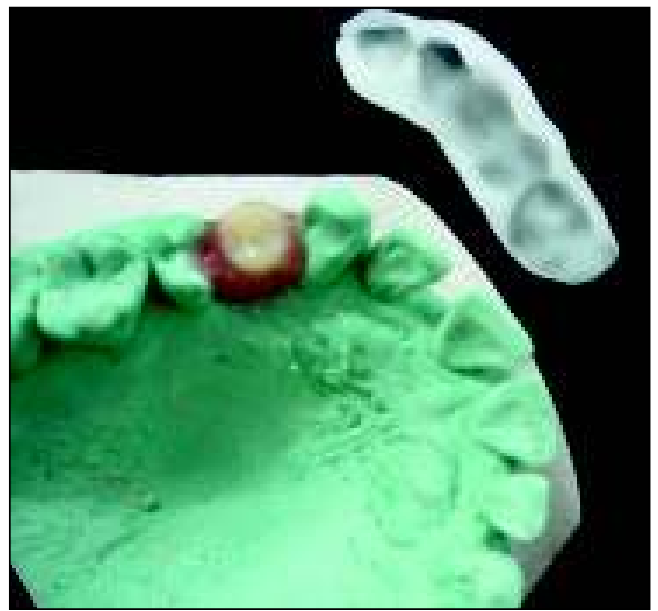

Matrix-completed

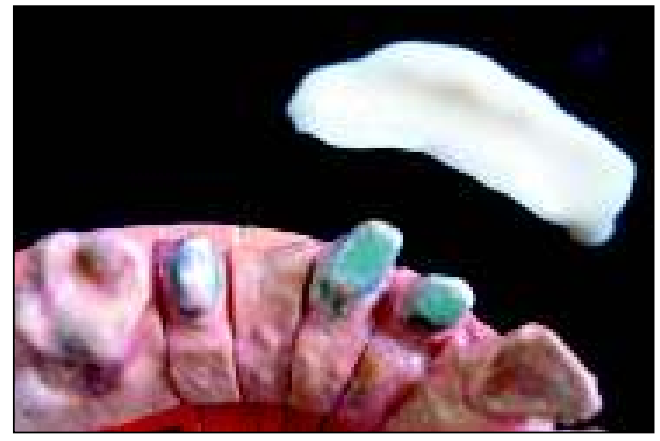

Loaded matrix on the prepared abutments

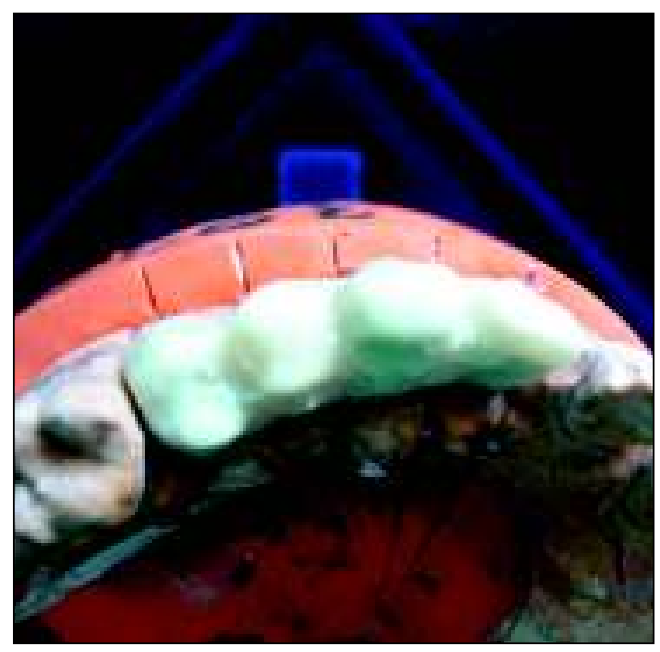

Provisional restoration - after trimming(before polishing) 
It can also be used in a fractured tooth after it is modified with rope wax. If more bulk is required around the cervical area of the restoration, all that has to be done is to spring open the matrix buccally and lingually.

Extended advantage of this material is that it can also be used to form provisional laminates for anterior teeth with light cure acrylics.

\section{References:}

1. Malone WFP, Koth DL, Cavazos Jr E, Kaiser DA, Morgano SM. Tylman's Theory and Practice of Fixed Prosthodontics, $8^{\text {th }}$ edition, Ishiyaku EuroAmerica, Inc. Publishers. Tokyo. St.Louis. Chapter 11, pg 255-271.

2. Psichogios PC, Monaco EJ. Expedient direct approach for esthetic and functional provisional restorations. J Prosthet.Dent 2003;89:319-22

3. Haddix JE. A technique for visible light-cured provisional restorations. J Prosthet Dent 1988;59:512-14

4. Savabi O, NejatiDanesh F. A method for fabrication of temporary restoration on solid abutment of ITI implants. J Prosthet Dent 2003;89:419

5. Erkut S. Provisional restorations for occlusal inlays using an orthodontic ligature tie. J Prosthet dent 2005;93:584-5

6. Rosenstiel SF, Land MF, Fujimoto J. Contemporary Fixed Prosthodontics. Second Edition. M osby. Pg.329-336

7. Dykema RW, Goodacre CJ, Phillips RW. Johnston's M odern Practice in Fixed Prosthodontics. Fourth Edition. Igaku-Shoin/Saunders International Edition. 1986. pages 78-86

8. www.advantagedentalinc.com 